\title{
Two-Higgs-doublet model fits with HEPfit
}

\section{Otto Eberhardt*}

Instituto de Física Corpuscular, Universitat de València - Consejo Superior de Investigaciones

Científicas, Parc Científic, E-46980 Paterna, Valencia, Spain

E-mail: otto.eberhardt@ific.uv.es

The Two-Higgs-doublet model (2HDM) is one of the most studied extensions of the Standard Model. Like the other popular "New Physics" models, it gets more and more constrained by recent experimental progress, especially by the LHC data. For the 2HDM types I and II with a softly broken $\mathbb{Z}_{2}$ symmetry, I present updated results of global analyses obtained with the open-source HEPfit code, emphasizing the impact of the pre-EPS-HEP 2017 LHC data. Furthermore, I discuss the status of implementation into HEPfit of 2 HDM's beyond the conventional $\mathbb{Z}_{2}$ symmetric types.

The European Physical Society Conference on High Energy Physics

5-12 July

Venice, Italy

${ }^{*}$ Speaker. 


\section{Introduction}

In spite of no direct indication for physics beyond the Standard Model of particle physics at the LHC, there are many good experimental and theoretical reasons to assume that the Standard Model (SM) is an incomplete description of our world. Lacking distinct measurements of new phenomena, two things become crucial for the analyses of SM extensions: The precise understanding of the SM as well as the consistent combination of all information on possible indirect signs of "New Physics" we can gather. In the following, I will present such an analysis for one of the most popular models that extend the SM, the Two-Higgs-Doublet model. It adds a second Higgs doublet to the SM particle content. In the past, many different constraints on this model have been explored; recently, the discovery of the $125 \mathrm{GeV}$ scalar $[1,2]$ as well as the absence of a second scalar resonance at the LHC have put strong bounds on the existence of a second Higgs doublet. Here, I will quantify these bounds in the light of updated LHC data, performing global fits to the Two-Higgs-Doublet models with a softly broken $\mathbb{Z}_{2}$ symmetry ( $2 \mathrm{HDM}$ ) of type I and II. Before going into detail, I will give an introduction to the fitting framework HEPfit, which also guarantees the consistent treatment of the SM part at the best precision available.

\section{HEPfit}

As statistical setup for the global 2HDM fits I use the open-source C++ code HEPfit [3], which is linked to the Bayesian Analysis Toolkit (BAT) [4]. HEPfit calculates flavour and Higgs observables as well as electroweak Z-pole observables, most of them at the best known precision. It can be linked to other programs as a library, but it also comes with an interface to BAT and can be used to perform global fits in the SM and several of its extensions (see also the other HEPfit contributions at EPS-HEP 2017 [5, 6, 7]). The release of the first fully documented HEPfit version is planned in the near future.

\section{The 2HDM}

The most general formulation of Two-Higgs-Doublet models $[8,9,10]$ is characterized by the Higgs potential

$$
\begin{aligned}
V_{H}^{2 \mathrm{HDM}}= & m_{11}^{2} \Phi_{1}^{\dagger} \Phi_{1}+m_{22}^{2} \Phi_{2}^{\dagger} \Phi_{2}-\left(m_{12}^{2} \Phi_{1}^{\dagger} \Phi_{2}+\text { H.c. }\right) \\
& +\frac{\lambda_{1}}{2}\left(\Phi_{1}^{\dagger} \Phi_{1}\right)^{2}+\frac{\lambda_{2}}{2}\left(\Phi_{2}^{\dagger} \Phi_{2}\right)^{2}+\lambda_{3}\left(\Phi_{1}^{\dagger} \Phi_{1}\right)\left(\Phi_{2}^{\dagger} \Phi_{2}\right)+\lambda_{4}\left(\Phi_{1}^{\dagger} \Phi_{2}\right)\left(\Phi_{2}^{\dagger} \Phi_{1}\right) \\
& +\left[\frac{\lambda_{5}}{2}\left(\Phi_{1}^{\dagger} \Phi_{2}\right)^{2}+\lambda_{6}\left(\Phi_{1}^{\dagger} \Phi_{1}\right)\left(\Phi_{1}^{\dagger} \Phi_{2}\right)+\lambda_{7}\left(\Phi_{2}^{\dagger} \Phi_{2}\right)\left(\Phi_{1}^{\dagger} \Phi_{2}\right)+\text { H.c. }\right]
\end{aligned}
$$

where $\Phi_{1}$ and $\Phi_{2}$ are the two Higgs doublets.

The corresponding Yukawa Lagrangian reads 


$$
\begin{aligned}
\mathscr{L}_{\text {Yukawa }}=-\sum_{j, k=1}^{3}[ & Y_{j k}^{d, 1}\left(\bar{Q}_{j} \Phi_{1}\right) d_{k}+Y_{j k}^{d, 2}\left(\bar{Q}_{j} \Phi_{2}\right) d_{k} \\
& +Y_{j k}^{u, 1}\left(\bar{Q}_{j} \mathrm{i} \sigma_{2} \Phi_{1}^{*}\right) u_{k}+Y_{j k}^{u, 2}\left(\bar{Q}_{j} \mathrm{i} \sigma_{2} \Phi_{2}^{*}\right) u_{k} \\
& \left.+Y_{j k}^{\ell, 1}\left(\bar{L}_{j} \Phi_{1}\right) \ell_{k}+Y_{j k}^{\ell, 2}\left(\bar{L}_{j} \Phi_{2}\right) \ell_{k}+\text { H.c. }\right],
\end{aligned}
$$

with the left-handed fermion fields $Q$ and $L$ and the right-handed fermion fields $u, d$ and $\ell$.

While the HEPfit collaboration is working at an implementation of the most general model into HEPfit, I will focus here on the case without explicitly broken $\mathbb{Z}_{2}$ symmetry. This implies $\lambda_{6}=\lambda_{7}=Y^{u, 1}=0$ and either $Y^{d, 1}=Y^{\ell, 1}=0$ (type I) or $Y^{d, 2}=Y^{\ell, 2}=0$ (type II). ${ }^{1}$ For an example of a 2HDM fit to a more general Yukawa sector with HEPfit, see [7]. I furthermore assume that all couplings in $V_{H}^{2 \mathrm{HDM}}$ are real and that the $125 \mathrm{GeV}$ scalar is the lightest $2 \mathrm{HDM}$ scalar $h$. The other physical Higgs particles are the neutral scalar $H$, the neutral pseudoscalar $A$ and the charged scalars $H^{ \pm}$. In the fits, I assume that their masses as well as the soft $\mathbb{Z}_{2}$ breaking scale $\left|m_{12}\right|$ are below 1.5 $\mathrm{TeV}$. Apart from these masses, the $2 \mathrm{HDM}$ is defined by the two mixing angles $\alpha$ and $\beta$ between these scalars, instead of which I will use $\tan \beta$ and $\beta-\alpha$. The SM parameters are fixed to their best fit values [12].

\section{Constraints}

As mentioned before, I want to emphasize the impact of the LHC measurements on the 2HDM parameter space. They can be divided into the signal strengths of the $125 \mathrm{GeV}$ resonance $h$ and the searches for heavier scalars. For both, I use all available data from the $7+8 \mathrm{TeV}$ run and the $13 \mathrm{TeV}$ run which was made public before the EPS-HEP 2017 conference: signal strengths of $h$ decaying to $\gamma \gamma, b b, \tau \tau, \mu \mu, W W$ and $Z Z[13,14,15,16,17,18,19,20,21,22,23,24]$ and the search for heavy neutral resonances in decays to $b b, \tau \tau, \gamma \gamma, Z \gamma, Z Z, W W, h h$ and $h Z$ $[25,26,27,28,29,30,31,32,33,34,35,36,37,38,39,40,41,42,43,44,45,46,47,48,49,50$, $51,52,53,21,54,55,56,57,58,59,60,61,62,63]$ as well as the search for charged scalars with the final states $\tau v$ and $t b[64,65,66,67,68,69,70]$. The details of the implementation of these observables into HEPfit can be found in [71, 11]; here, I assume that the observed upper limits on the cross sections are identical with the expected ones, given that almost everywhere the two are compatible at the $2 \sigma$ level.

On top of the LHC data, I apply a conservative choice for the theoretical constraints: In order to require that the vacuum is stable, I need to guarantee that $V_{H}^{2 \mathrm{HDM}}$ is bounded from below [72] and that the electroweak minimum is the global minimum [73]. Moreover, I demand that the eigenvalues of the scattering matrix of two-scalar-to-two-scalar scattering processes do not exceed 1 in magnitude [74], and that the next-to-leading order contribution to these eigenvalues is not larger than its leading order value [75, 71]. Finally, I combine the mentioned bounds with the remaining relevant constraints: The 2HDM should be in agreement with electroweak precision data, so I use the latest HEPfit values [12, 6] for the Peskin-Takeuchi pseudo-observables $S, T$ and $U[76,77,78]$. I also include the two most relevant flavour observables to the fit, namely $\mathscr{B}(b \rightarrow s \gamma)$ and $\Delta m_{B_{s}}$ [79, 80, 81, 82].

\footnotetext{
${ }^{1}$ For the cases with $Y^{d, 1}=Y^{\ell, 2}=0$ or $Y^{d, 2}=Y^{\ell, 1}=0$ I refer to [11].
} 


\section{Fit results}

To start with, I discuss the effect the $h$ signal strengths have on the 2HDM parameters. Since the tree-level couplings of $h$ to fermions and gauge bosons only depend on the 2HDM angles, it is obvious to study the $\beta-\alpha$ vs. $\tan \beta$ plane. For both types, these planes are shown in Figure 1 with the single contributions of all relevant $h$ decays as well as their combination. While $\tan \beta$ can have any value between 0.3 and 30, the difference between $\beta$ and $\alpha$ is forced to be close to the socalled alignment limit of $\pi / 2$ for which the $h$ couplings become SM-like. The maximal deviation of this value depends on $\tan \beta$ and the 2HDM type and is smaller compared to the fits to data from the $7+8 \mathrm{TeV}$ run of the LHC (see e.g. [83, 84]). In the low $\tan \beta$ range, the $\gamma \gamma$ signal strengths prevail in type I, whereas for $\tan \beta>8$ the $h \rightarrow Z Z$ measurements are the strongest. In type II, the most stringent bounds come from the $Z Z$ and $W W$ signal strengths. The tree-level coupling of the $h$ boson to massive gauge bosons is type independent, but these constraints also depend on the fermion couplings in the $h$ production and decay width; that is why the bounds are much stronger in type II. For the latter, it is also worth mentioning that the so-called wrong-sign Yukawa coupling solution is only compatible with all signal strengths in a very small region around $\tan \beta=3.2$ and $\beta-\alpha=1$.
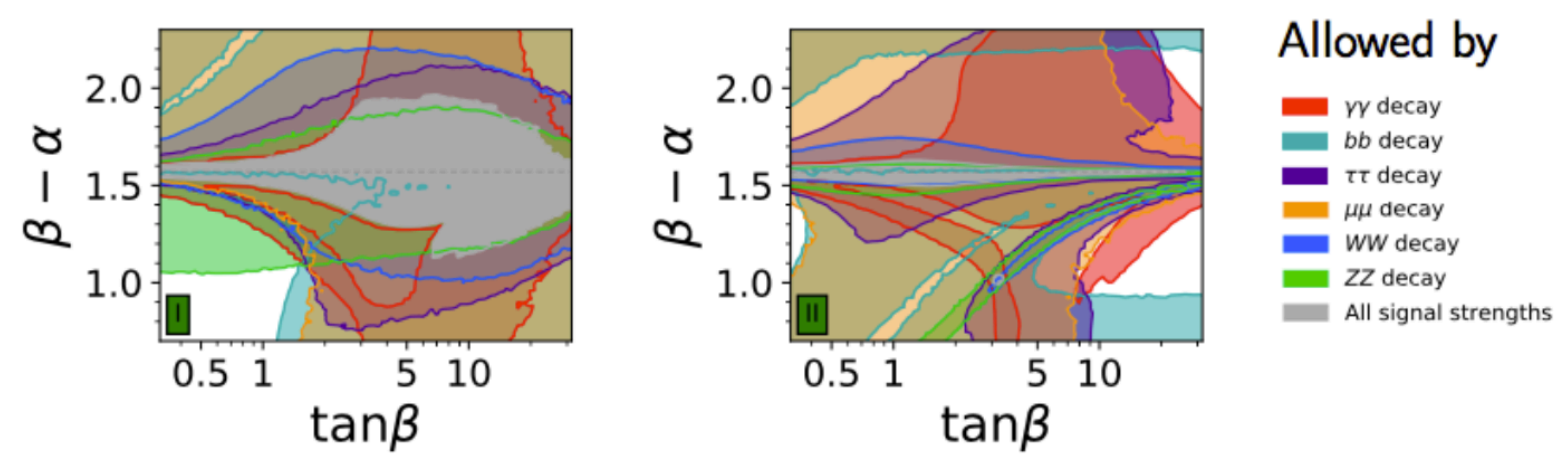

Figure 1: $95 \%$ allowed regions in the $\beta-\alpha$ vs. $\tan \beta$ plane. The coloured contours are allowed by the $h$ decays to $\gamma \gamma, b b, \tau \tau, \mu \mu, W W$ and $Z Z$, respectively (see the legend). The grey area results from the combination of all signal strengths. In the left panel, the type I fit result is displayed and in the right panel the one from the type II fit.

In Figure 2 I show the combination of all signal strengths transferred to the $\beta-\alpha$ vs. $m_{H}$ plane; it is independent of the heavy Higgs mass. I confront it with the regions disfavoured by all heavy Higgs searches, which mainly constrain $H$ masses below $1 \mathrm{TeV}$, and the scenarios excluded by the theoretical bounds, which for $m_{H}$ above $600 \mathrm{GeV}$ push the 2HDM towards the alignment limit. While both, the heavy Higgs searches and the theoretical constraints hardly have an effect stronger than the signal strengths in this plane for type II, they are more relevant in type I, where the impact of the signal strengths is weaker. Finally, combining the LHC and theory constraints with the ones from flavour and Z-pole physics, one obtains the strips within the black contours. In both types, the global fit to all constraints only allows small deviations from the alignment limit; the type II $H$ mass additionally gets a lower bound of around $750 \mathrm{GeV}$ if one simultaneously fits the lower bound on the charged Higgs mass from $b \rightarrow s \gamma$ transitions with the electroweak precision observables and the theory constraints (see also [71, 11]). 

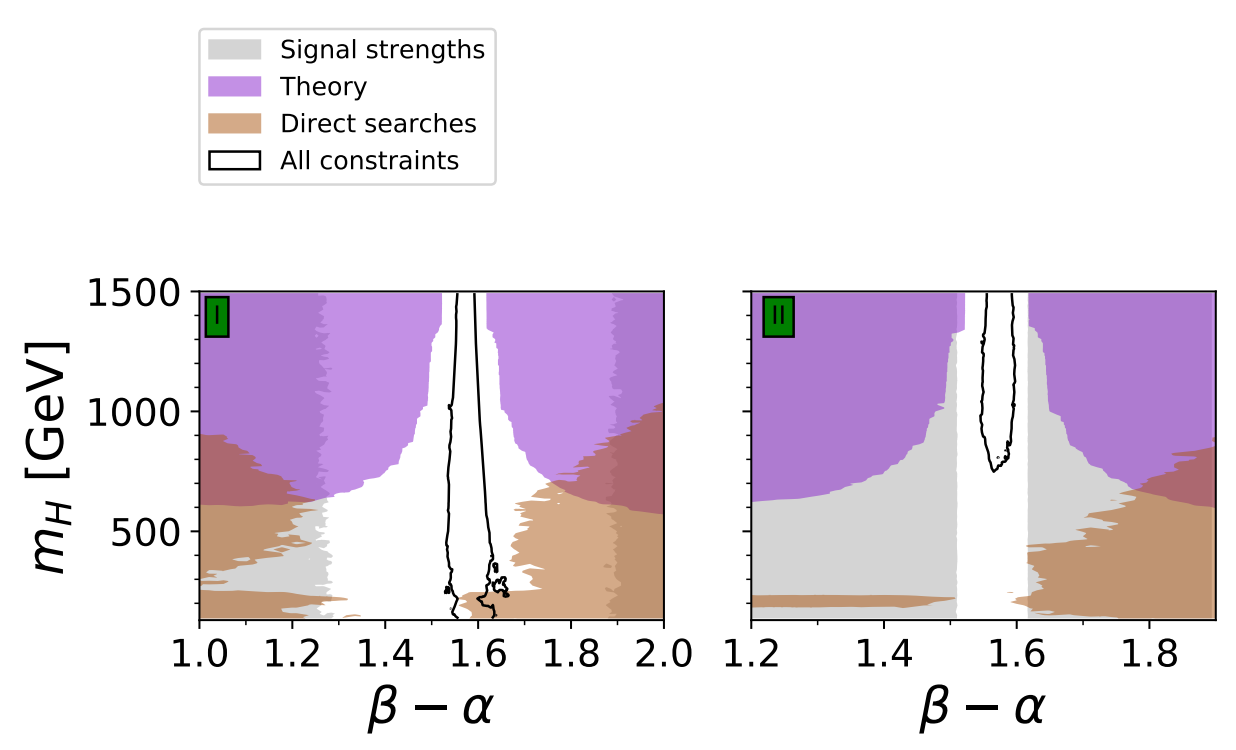

Figure 2: $95 \%$ excluded regions in the $m_{H}$ vs. $\beta-\alpha$ planes of type I (left) and II (right). The exclusions stem from all signal strengths (grey), all heavy Higgs searches (orange), theoretical bounds (purple). The region within the black contour is allowed after the combination of all constraints.

\section{Summary}

After introducing the multi-purpose code HEPfit, I show its application to the 2HDM types I and II: I discuss the impact of the $h$ signal strengths and the searches for heavy Higgs bosons on the 2HDM parameter space. These measurements by the ATLAS and CMS collaborations yield strong bounds especially on the angle difference $\beta-\alpha$. With increasing data, it is being pushed more and more to the value of $\pi / 2$, for which the 125 Higgs resembles the SM Higgs. For even more up-to-date fits, also to the two remaining types of $\mathbb{Z}_{2}$ symmetry I have not discussed here, I refer to [11].

\section{Acknowledgments}

I thank Debtosh Chowdhury for helpful discussions. This work was supported by the Spanish Government and ERDF funds from the European Commission (Grants No. FPA2014-53631-C21-P and SEV-2014-0398).

\section{References}

[1] ATLAS collaboration, G. Aad et al., Observation of a new particle in the search for the Standard Model Higgs boson with the ATLAS detector at the LHC, Phys.Lett. B716 (2012) 1-29, [1207. 7214].

[2] CMS collaboration, S. Chatrchyan et al., Observation of a new boson at a mass of $125 \mathrm{GeV}$ with the CMS experiment at the LHC, Phys. Lett. B716 (2012) 30-61, [1207 . 7235]. 
[3] HEPFIT collaboration. HEP fit: a Code for the Combination of Indirect and Direct Constraints on High Energy Physics Models, in preparation. http://hepfit.roma1.infn.it.

[4] A. Caldwell, D. Kollar and K. Kroninger, BAT: The Bayesian Analysis Toolkit, Comput. Phys. Commun. 180 (2009) 2197-2209, [0 808 .2552].

[5] M. Valli, "A Systematic Study of Discriminators between New Physics and Standard Model in $b \rightarrow s$ transitions." Talk given at EPS-HEP 2017, https://indico.cern.ch/event/466934/contributions/2589682.

[6] L. Silvestrini, “The global electroweak fit: present status, constraints on new physics, and prospects at future colliders." Talk given at EPS-HEP 2017, https://indico.cern.ch/event/466934/contributions/2575364.

[7] A. Paul, "Flavour Physics meets Heavy Higgs Searches.” Talk given at EPS-HEP 2017, https://indico.cern.ch/event/466934/contributions/2586401.

[8] T. Lee, A Theory of Spontaneous T Violation, Phys.Rev. D8 (1973) 1226-1239.

[9] J. F. Gunion and H. E. Haber, The CP conserving two Higgs doublet model: The Approach to the decoupling limit, Phys. Rev. D67 (2003) 075019, [hep-ph/ 0207010 ].

[10] G. Branco, P. Ferreira, L. Lavoura, M. Rebelo, M. Sher et al., Theory and phenomenology of two-Higgs-doublet models, Phys.Rept. 516 (2012) 1-102, [110 6.0034$].$

[11] D. Chowdhury and O. Eberhardt, "Update of global Two-Higgs-Doublet model fits." in preparation, 2017.

[12] J. de Blas, M. Ciuchini, E. Franco, S. Mishima, M. Pierini, L. Reina et al., Electroweak precision observables and Higgs-boson signal strengths in the Standard Model and beyond: present and future, 1608.01509.

[13] ATLAS, CMS collaboration, G. Aad et al., Measurements of the Higgs boson production and decay rates and constraints on its couplings from a combined ATLAS and CMS analysis of the LHC pp collision data at $\sqrt{s}=7$ and $8 \mathrm{TeV}, 1606.02266$.

[14] ATLAS collaboration, Search for Higgs boson production via weak boson fusion and decaying to b $\bar{b}$ in association with a high-energy photon in the ATLAS detector, Tech. Rep.

ATLAS-CONF-2016-063, CERN, Geneva, Aug, 2016.

[15] ATLAS collaboration, Search for the Standard Model Higgs boson produced in association with top quarks and decaying into b $\bar{b}$ in pp collisions at $\sqrt{s}=13$ TeV with the ATLAS detector, Tech. Rep. ATLAS-CONF-2016-080, CERN, Geneva, Aug, 2016.

[16] ATLAS collaboration, Combined measurements of the Higgs boson production and decay rates in $H \rightarrow Z Z^{*} \rightarrow 4 \ell$ and $H \rightarrow \gamma \gamma$ final states using pp collision data at $\sqrt{s}=13 \mathrm{TeV}$ in the ATLAS experiment, Tech. Rep. ATLAS-CONF-2016-081, CERN, Geneva, Aug, 2016.

[17] ATLAS collaboration, Search for the Standard Model Higgs boson produced in association with a vector boson and decaying to a b $\bar{b}$ pair in pp collisions at $13 \mathrm{TeV}$ using the ATLAS detector, Tech. Rep. ATLAS-CONF-2016-091, CERN, Geneva, Aug, 2016.

[18] ATLAS collaboration, Measurements of the Higgs boson production cross section via Vector Boson Fusion and associated $W H$ production in the $W W^{*} \rightarrow \ell v \ell v$ decay mode with the ATLAS detector at $\sqrt{s}=13 \mathrm{TeV}$, Tech. Rep. ATLAS-CONF-2016-112, CERN, Geneva, Nov, 2016.

[19] CMS collaboration, Search for the standard model Higgs boson produced through vector boson fusion and decaying to bb with proton-proton collisions at $\operatorname{sqrt}(\mathrm{s})=13 \mathrm{TeV}$, Tech. Rep. CMS-PAS-HIG-16-003, CERN, Geneva, 2016. 
[20] CMS collaboration, Updated measurements of Higgs boson production in the diphoton decay channel at $\sqrt{s}=13 \mathrm{TeV}$ in pp collisions at CMS., Tech. Rep. CMS-PAS-HIG-16-020, CERN, Geneva, 2016.

[21] CMS collaboration, Measurements of properties of the Higgs boson and search for an additional resonance in the four-lepton final state at $\operatorname{sqrt}(s)=13 \mathrm{TeV}$, Tech. Rep. CMS-PAS-HIG-16-033, CERN, Geneva, 2016.

[22] CMS collaboration, Observation of the SM scalar boson decaying to a pair of $\tau$ leptons with the CMS experiment at the LHC, Tech. Rep. CMS-PAS-HIG-16-043, CERN, Geneva, 2017.

[23] CMS collaboration, Search for $\mathrm{t} \overline{\mathrm{t}} \mathrm{H}$ production in the $\mathrm{H} \rightarrow \mathrm{b} \overline{\mathrm{b}}$ decay channel with 2016 pp collision data at $\sqrt{s}=13 \mathrm{TeV}$, Tech. Rep. CMS-PAS-HIG-16-038, CERN, Geneva, 2016.

[24] CMS collaboration, Search for the associated production of a Higgs boson with a top quark pair in final states with a $\tau$ lepton at $\sqrt{s}=13 \mathrm{TeV}$, Tech. Rep. CMS-PAS-HIG-17-003, CERN, Geneva, 2017.

[25] ATLAS collaboration, G. Aad et al., Search for new resonances in $W \gamma$ and $Z \gamma$ final states in $p p$ collisions at $\sqrt{s}=8 \mathrm{TeV}$ with the ATLAS detector, Phys. Lett. B738 (2014) 428-447, [1407.8150].

[26] ATLAS collaboration, G. Aad et al., Search for Scalar Diphoton Resonances in the Mass Range 65-600 GeV with the ATLAS Detector in pp Collision Data at $\sqrt{s}=8 \mathrm{TeV}$, Phys. Rev. Lett. 113 (2014) 171801, [1407.6583].

[27] ATLAS collaboration, G. Aad et al., Search for neutral Higgs bosons of the minimal supersymmetric standard model in pp collisions at $\sqrt{s}=8 \mathrm{TeV}$ with the ATLAS detector, JHEP 11 (2014) 056, [1409.6064].

[28] ATLAS collaboration, G. Aad et al., Search for a high-mass Higgs boson decaying to a W boson pair in pp collisions at $\sqrt{s}=8 \mathrm{TeV}$ with the ATLAS detector, JHEP 01 (2016) 032, [1509.00389].

[29] ATLAS collaboration, G. Aad et al., Search for an additional, heavy Higgs boson in the $H \rightarrow Z Z$ decay channel at $\sqrt{s}=8 \mathrm{TeV}$ in pp collision data with the ATLAS detector, Eur. Phys. J. C76 (2016) 45, [1507.05930].

[30] ATLAS collaboration, G. Aad et al., Search for a CP-odd Higgs boson decaying to Zh in pp collisions at $\sqrt{s}=8 \mathrm{TeV}$ with the ATLAS detector, Phys. Lett. B744 (2015) 163-183, [1502.04478].

[31] ATLAS collaboration, G. Aad et al., Searches for Higgs boson pair production in the $h h \rightarrow b b \tau \tau, \gamma \gamma W W^{*}, \gamma \gamma b b, b b b b$ channels with the ATLAS detector, Phys. Rev. D92 (2015) 092004, [1509.04670].

[32] ATLAS collaboration, Search for new phenomena in the $Z(\rightarrow \ell \ell)+E_{\mathrm{T}}^{\text {miss }}$ final state at $\sqrt{s}=13 \mathrm{TeV}$ with the ATLAS detector, Tech. Rep. ATLAS-CONF-2016-056, CERN, Geneva, Aug, 2016.

[33] ATLAS collaboration, Search for diboson resonance production in the $\ell$ vq final state using pp collisions at $\sqrt{s}=13 \mathrm{TeV}$ with the ATLAS detector at the LHC, Tech. Rep. ATLAS-CONF-2016-062, CERN, Geneva, Aug, 2016.

[34] ATLAS collaboration, Search for scalar diphoton resonances with $15.4 \mathrm{fb}^{-1}$ of data collected at $\sqrt{s}=13 \mathrm{TeV}$ in 2015 and 2016 with the ATLAS detector, Tech. Rep. ATLAS-CONF-2016-059, CERN, Geneva, Aug, 2016.

[35] ATLAS collaboration, Search for Minimal Supersymmetric Standard Model Higgs Bosons H/A in the $\tau \tau$ final state in up to $13.3 \mathrm{fb}^{-1}$ of pp collisions at $\sqrt{\mathrm{s}}=13 \mathrm{TeV}$ with the ATLAS Detector, Tech. Rep. ATLAS-CONF-2016-085, CERN, Geneva, Aug, 2016. 
[36] ATLAS collaboration, Search for a high-mass Higgs boson decaying to a pair of W bosons in pp collisions at $\sqrt{s}=13 \mathrm{TeV}$ with the ATLAS detector, Tech. Rep. ATLAS-CONF-2016-074, CERN, Geneva, Aug, 2016.

[37] ATLAS collaboration, Search for new resonances decaying to a $Z$ boson and a photon in $13.3 \mathrm{fb}^{-1}$ of pp collisions at $\sqrt{s}=13 \mathrm{TeV}$ with the ATLAS detector, Tech. Rep. ATLAS-CONF-2016-044, CERN, Geneva, Aug, 2016.

[38] ATLAS collaboration, Searches for heavy ZZ and ZW resonances in the llqq and vvqq final states in pp collisions at sqrt(s) =13 TeV with the ATLAS detector, Tech. Rep. ATLAS-CONF-2016-082, CERN, Geneva, Aug, 2016.

[39] ATLAS collaboration, Study of the Higgs boson properties and search for high-mass scalar resonances in the $H \rightarrow Z Z^{*} \rightarrow 4 \ell$ decay channel at $\sqrt{s}=13$ TeV with the ATLAS detector, Tech. Rep. ATLAS-CONF-2016-079, CERN, Geneva, Aug, 2016.

[40] ATLAS collaboration, Search for Higgs boson pair production in the final state of $\gamma \gamma W W^{*}(\rightarrow l v j j)$ using $13.3 \mathrm{fb}^{-1}$ of pp collision data recorded at $\sqrt{\mathrm{s}}=13 \mathrm{TeV}$ with the ATLAS detector, Tech. Rep. ATLAS-CONF-2016-071, CERN, Geneva, Aug, 2016.

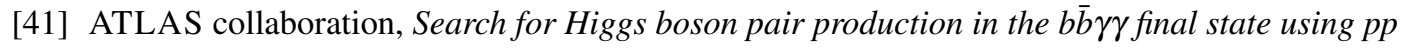
collision data at $\sqrt{s}=13 \mathrm{TeV}$ with the ATLAS detector, Tech. Rep. ATLAS-CONF-2016-004, CERN, Geneva, Mar, 2016.

[42] ATLAS collaboration, Search for a CP-odd Higgs boson decaying to Zh in pp collisions at $\sqrt{s}=13 \mathrm{TeV}$ with the ATLAS detector, Tech. Rep. ATLAS-CONF-2016-015, CERN, Geneva, Mar, 2016.

[43] ATLAS collaboration, Search for pair production of Higgs bosons in the $b \bar{b} b \bar{b}$ final state using proton-proton collisions at $\sqrt{s}=13 \mathrm{TeV}$ with the ATLAS detector, Tech. Rep.

ATLAS-CONF-2016-017, CERN, Geneva, Mar, 2016.

[44] CMS collaboration, V. Khachatryan et al., Search for a Higgs Boson in the Mass Range from 145 to 1000 GeV Decaying to a Pair of W or Z Bosons, JHEP 10 (2015) 144, [1504 . 00936 ].

[45] CMS collaboration, V. Khachatryan et al., Search for a pseudoscalar boson decaying into a $Z$ boson and the $125 \mathrm{GeV}$ Higgs boson in $\ell^{+} \ell b \bar{b}$ final states, Phys. Lett. B748 (2015) 221-243, [1504.04710].

[46] CMS collaboration, V. Khachatryan et al., Searches for a heavy scalar boson H decaying to a pair of $125 \mathrm{GeV}$ Higgs bosons hh or for a heavy pseudoscalar boson A decaying to Zh, in the final states with $h \rightarrow \tau \tau$, Phys. Lett. B755 (2016) 217-244, [1510.01181].

[47] CMS collaboration, V. Khachatryan et al., Search for Neutral MSSM Higgs Bosons Decaying into A Pair of Bottom Quarks, JHEP 11 (2015) 071, [1506.08329].

[48] CMS collaboration, V. Khachatryan et al., Search for resonant pair production of Higgs bosons decaying to two bottom quark-antiquark pairs in proton-proton collisions at $8 \mathrm{TeV}$, Phys. Lett. B749 (2015) 560-582, [1503.04114].

[49] CMS collaboration, V. Khachatryan et al., Search for two Higgs bosons in final states containing two photons and two bottom quarks in proton-proton collisions at 8 TeV, Phys. Rev. D94 (2016) 052012, [1603.06896].

[50] CMS collaboration, Search for additional neutral Higgs bosons decaying to a pair of tau leptons in pp collisions at $\sqrt{s}=7$ and $8 \mathrm{TeV}$, Tech. Rep. CMS-PAS-HIG-14-029, CERN, Geneva, 2015. 
[51] CMS collaboration, Search for scalar resonances in the 200-1200 GeV mass range decaying into a $Z$ and a photon in pp collisions at $\sqrt{s}=8 \mathrm{TeV}$, Tech. Rep. CMS-PAS-HIG-16-014, CERN, Geneva, 2016.

[52] CMS collaboration, Search for high-mass resonances in $\mathrm{Z}(\mathrm{q} \overline{\mathrm{q}}) \gamma$ final state in pp collisions at $\sqrt{s}=13 \mathrm{TeV}$ with $12.9 \mathrm{fb}^{-1}$, Tech. Rep. CMS-PAS-EXO-16-035, CERN, Geneva, 2016.

[53] CMS collaboration, Search for resonant production of high mass photon pairs using $12.9 \mathrm{fb}^{-1}$ of proton-proton collisions at $\sqrt{s}=13 \mathrm{TeV}$ and combined interpretation of searches at 8 and $13 \mathrm{TeV}$, Tech. Rep. CMS-PAS-EXO-16-027, CERN, Geneva, 2016.

[54] CMS collaboration, Search for high mass Higgs to WW with fully leptonic decays using 2015 data, Tech. Rep. CMS-PAS-HIG-16-023, CERN, Geneva, 2016.

[55] CMS collaboration, Search for resonant Higgs boson pair production in the $\mathrm{b} \overline{\mathrm{b}} \tau^{+} \tau^{-}$final state using 2016 data, Tech. Rep. CMS-PAS-HIG-16-029, CERN, Geneva, 2016.

[56] CMS collaboration, Search for a narrow heavy decaying to bottom quark pairs in the $13 \mathrm{TeV}$ data sample, Tech. Rep. CMS-PAS-HIG-16-025, CERN, Geneva, 2016.

[57] CMS collaboration, Search for high-mass resonances in $Z \gamma \rightarrow e^{+} e^{-} \gamma / \mu^{+} \mu^{-} \gamma$ final states in proton-proton collisions at $\sqrt{s}=13 \mathrm{TeV}$, Tech. Rep. CMS-PAS-EXO-16-034, CERN, Geneva, 2016.

[58] CMS collaboration, Search for resonant Higgs boson pair production in the $\mathrm{b} \bar{b} l v l v$ final state at $\sqrt{s}=13 \mathrm{TeV}$, Tech. Rep. CMS-PAS-HIG-16-011, CERN, Geneva, 2016.

[59] CMS collaboration, Search for a neutral MSSM Higgs boson decaying into $\tau \tau$ with $12.9 \mathrm{fb}^{-1}$ of data at $\sqrt{s}=13 \mathrm{TeV}$, Tech. Rep. CMS-PAS-HIG-16-037, CERN, Geneva, 2016.

[60] CMS collaboration, Search for resonant pair production of Higgs bosons decaying to two bottom quark-antiquark pairs in proton-proton collisions at $13 \mathrm{TeV}$, Tech. Rep. CMS-PAS-HIG-16-002, CERN, Geneva, 2016.

[61] CMS collaboration, Search for $H($ bb)H(gammagamma) decays at 13TeV, Tech. Rep. CMS-PAS-HIG-16-032, CERN, Geneva, 2016.

[62] CMS collaboration, Model independent search for Higgs boson pair production in the $\mathrm{b} \overline{\mathrm{b}} \tau^{+} \tau^{-}$final state, Tech. Rep. CMS-PAS-HIG-15-013, CERN, Geneva, 2016.

[63] CMS collaboration, Search for new diboson resonances in the dilepton + jets final state at $\sqrt{s}=13 \mathrm{TeV}$ with 2016 data, Tech. Rep. CMS-PAS-HIG-16-034, CERN, Geneva, 2017.

[64] ATLAS collaboration, G. Aad et al., Search for charged Higgs bosons decaying via $H^{ \pm} \rightarrow \tau^{ \pm} v$ in fully hadronic final states using pp collision data at $\sqrt{s}=8 \mathrm{TeV}$ with the ATLAS detector, JHEP 03 (2015) 088, [1412.6663].

[65] CMS collaboration, V. Khachatryan et al., Search for a charged Higgs boson in pp collisions at $\sqrt{s}=8 \mathrm{TeV}$, JHEP 11 (2015) 018, [1508.07774].

[66] ATLAS collaboration, G. Aad et al., Search for charged Higgs bosons in the $H^{ \pm} \rightarrow$ tb decay channel in pp collisions at $\sqrt{s}=8 \mathrm{TeV}$ using the ATLAS detector, JHEP 03 (2016) 127, [1512. 03704 ].

[67] ATLAS collaboration, Search for charged Higgs bosons in the $\tau+$ jets final state using $14.7 \mathrm{fb}^{-1}$ of pp collision data recorded at $\sqrt{s}=13 \mathrm{TeV}$ with the ATLAS experiment, Tech. Rep. ATLAS-CONF-2016-088, CERN, Geneva, Aug, 2016.

[68] CMS collaboration, Search for charged Higgs bosons with the $\mathrm{H}^{ \pm} \rightarrow \tau^{ \pm} v_{\tau}$ decay channel in the fully hadronic final state at $\sqrt{s}=13 \mathrm{TeV}$, Tech. Rep. CMS-PAS-HIG-16-031, CERN, Geneva, 2016. 
[69] ATLAS collaboration, Search for charged Higgs bosons in the $H^{ \pm} \rightarrow$ tb decay channel in pp collisions at $\sqrt{s}=13 \mathrm{TeV}$ using the ATLAS detector, Tech. Rep. ATLAS-CONF-2016-089, CERN, Geneva, Aug, 2016.

[70] ATLAS collaboration, Search for new phenomena in $t \bar{t}$ final states with additional heavy-flavour jets in pp collisions at $\sqrt{s}=13 \mathrm{TeV}$ with the ATLAS detector, Tech. Rep. ATLAS-CONF-2016-104, CERN, Geneva, Sep, 2016.

[71] V. Cacchio, D. Chowdhury, O. Eberhardt and C. W. Murphy, Next-to-leading order unitarity fits in Two-Higgs-Doublet models with soft $\mathbb{Z}_{2}$ breaking, JHEP 11 (2016) 026, [1609.01290].

[72] N. G. Deshpande and E. Ma, Pattern of Symmetry Breaking with Two Higgs Doublets, Phys. Rev. D18 (1978) 2574.

[73] A. Barroso, P. M. Ferreira, I. P. Ivanov and R. Santos, Metastability bounds on the two Higgs doublet model, JHEP 06 (2013) 045, [1303.5098].

[74] I. F. Ginzburg and I. P. Ivanov, Tree-level unitarity constraints in the most general 2HDM, Phys. Rev. D72 (2005) 115010, [hep-ph/ 0508020$]$.

[75] B. Grinstein, C. W. Murphy and P. Uttayarat, One-loop corrections to the perturbative unitarity bounds in the CP-conserving two-Higgs doublet model with a softly broken $\mathbb{Z}_{2}$ symmetry, JHEP 06 (2016) 070, [1512.04567].

[76] M. E. Peskin and T. Takeuchi, A New constraint on a strongly interacting Higgs sector, Phys. Rev. Lett. 65 (1990) 964-967.

[77] M. E. Peskin and T. Takeuchi, Estimation of oblique electroweak corrections, Phys. Rev. D46 (1992) 381-409.

[78] H. E. Haber, Introductory low-energy supersymmetry, in Theoretical Advanced Study Institute (TASI 92): From Black Holes and Strings to Particles Boulder, Colorado, June 3-28, 1992, 1993. hep-ph/9306207.

[79] M. Misiak et al., Updated NNLO QCD predictions for the weak radiative B-meson decays, Phys. Rev. Lett. 114 (2015) 221801, [1503.01789].

[80] C. Q. Geng and J. N. Ng, Charged-Higgs-boson effect in $B_{d}^{0}-\bar{B}_{d}^{0}$ mixing, $K \rightarrow \pi v \bar{v}$ decay, and rare decays of B mesons, Phys. Rev. D38 (1988) 2857.

[81] O. Deschamps, S. Descotes-Genon, S. Monteil, V. Niess, S. T'Jampens and V. Tisserand, The Two Higgs Doublet of Type II facing flavour physics data, Phys. Rev. D82 (2010) 073012, [0 907.5135$].$

[82] Y. Amhis et al., Averages of b-hadron, c-hadron, and $\tau$-lepton properties as of summer 2016, 1612.07233.

[83] T. Peiffer, "Update of the Global Electroweak Fit by Gfitter.” Talk given at EPS-HEP 2017, https://indico.cern.ch/event/466934/contributions/2575352.

[84] D. Chowdhury and O. Eberhardt, Global fits of the two-loop renormalized Two-Higgs-Doublet model with soft $Z_{2}$ breaking, JHEP 11 (2015) 052, [1503.08216]. 